\title{
ON THE INERTIA GROUP OF A PRODUCT OF SPHERES
}

\author{
BY \\ REINHARD SCHULTZ
}

\begin{abstract}
In this paper it is proved that the smooth connected sum of a product of ordinary spheres with an exotic combinatorial sphere is never diffeomorphic to the original product. This result is extended and compared to certain related examples.
\end{abstract}

Before stating the result which provides our point of departure, we recall some standard notation. If $M^{n}$ is a closed smooth oriented $n$-manifold, the inertia group $I(M)$ consists of all exotic $n$-spheres $\Sigma$ such that $M \# \Sigma$ is orientation-preservingly diffeomorphic to $M$. Then in the first section of this paper we shall prove the following result:

THEOREM A. If $n \geqq 5$ and $M$ is a product of ordinary spheres, then $I(M)=0$.

The special case where $M$ is a product of two ordinary spheres was proved independently by DeSapio [6], Kawakubo [12], and the author [26]. Actually, a weaker version of Theorem A may be derived almost trivially by means of framed cobordism [14], and our proof may be considered an example of the relative difficulty of computing the intersection $I\left(M^{n}\right) \cap \partial P_{n+1}$ in general.

In the second section we discuss some generalizations of Theorem $\mathrm{A}$, and in 2.3 it is shown that the methods of $\$ 1$ generalize in a nonempty manner. We are led to conjecture the following result, which is proved in $\$ 4$ :

THEOREM C. Let $P^{k}$ be a product of ordinary spheres, and let $\Sigma^{n}$ be a homotopy sphere $(n \geqq 5)$. Then the inertia group of $\Sigma^{n} \times P^{k}$ is equal to the inertia group of $\Sigma^{n} \times S^{k}$.

This result is first proved for $P^{k}=T^{k}$, the $k$-dimensional torus, in $\S 3$. That section also contains a diffeomorphism classification theorem for all smooth manifolds homeomorphic to $S^{n} \times T^{k}$. The result (Theorem B) may be interpreted as an analog of the classification of smooth manifolds homeomorphic to $S^{n} \times S^{k}$, particularly as formulated in [17, Proposition 5.7].

RemarK. Our methods may be applied to determine whether any two given smoothings of a product of several spheres are diffeomorphic, but any closed

Received by the editors March 4, 1970.

AMS 1969 subject classifications. Primary 5710, 5720; Secondary 5542, 5731.

Key words and phrases. Inertia group, homotopy sphere, fiber homotopy equivalence, self-equivalence group, automorphisms of homology, surgery, smoothings of PL manifolds, homotopy smoothings, normal invariants, homotopy composition.

Copyright (C) 1971, American Mathematical Society 
formulas along the lines of [26, p. 187] would necessarily be quite involved and very tedious to express.

\section{Proof of Theorem A.}

1.1. The inertia group $I(M)$ was defined in the first paragraph of this paper. If $\Sigma$ is any topological sphere, let $i: M \# \Sigma \rightarrow M$ be the obvious homeomorphism. Then the subgroup $I_{0}(M) \subseteq I(M)$ will consist of all homotopy spheres $\Sigma$ such that there is an orientation-preserving diffeomorphism $h: M \# \Sigma \rightarrow M$ which induces the same maps in homology and fundamental groups as the mapping $i$ (compare [27, p. 82]).

REMARK. In general, $I_{0}(M) \neq I(M)$. The simplest examples known to the author occur when $M$ is a 9-dimensional fake torus; it may be chosen to be either combinatorially equivalent or inequivalent to $T^{9}$ as one prefers.

1.2. Proposition. Let $M$ be a closed smooth $n$-manifold $(n \geqq 7)$ which embeds in $R^{n+1}$. Then $I_{0}(M)=0$.

If $M$ is a product of the spheres $S_{1}, \ldots, S_{k}$ where the dimension of $S_{i}$ is $d(i)$, then there is a standard embedding of $R^{d(1)+1} \times S_{2}$ into $R^{d(1)+d(2)+1}$. Using such standard inclusion maps we get a chain of inclusions

$$
\begin{aligned}
M & =S_{1} \times S_{2} \times S_{3} \times \cdots \times S_{k} \subseteq R^{d(1)+d(2)+1} \times S_{3} \times \cdots \times S_{k} \\
& \subseteq R^{d(1)+d(2)+d(3)+1} \times \cdots \times S_{k} \\
& \subseteq R^{d(1)+\cdots+d(k-1)+1} \times S_{k} \subseteq R^{d(1)+\cdots+d(k)+1}
\end{aligned}
$$

This well-known argument is the one used to great advantage in NovikovSiebenmann splitting theory (compare [3]).

Proof. Without loss of generality, $M$ is contained in the unit disk $D^{n+1}$. Then $D^{n+1}$ is the union of two manifolds $V$ and $W$ such that $\partial V=M, \partial W=S^{n} \cup(-M)$, and $V \cap W=M$. Suppose now that $h: M \# \Sigma \rightarrow M$ is a diffeomorphism with the appropriate algebraic properties. Let $W_{1}$ be the cobordism formed by adding $\Sigma$ to both ends of $\partial W[26,1.1]$, and form the manifold $X=W_{1} \cup_{h} V$. By construction, $\partial X=\Sigma$. On the other hand, by a standard argument involving van Kampen's theorem, Mayer-Vietoris sequences, and the Hurewicz theorem it follows that $X$ is contractible and hence $\Sigma=S^{n}$.

REMARK. The quotient $I(M) / I_{0}(M)$ is a subquotient of the group Aut $H_{*}(M)$ of automorphisms of the homology groups of $M$. Explicitly, $I / I_{0} \cong A / B$, where $A \subseteq$ Aut $H_{*}(M)$ consists of all automorphisms $\varphi$ such that $\varphi=\left(i^{-1} h\right)_{*}$ for some almost diffeomorphism $h$ and $B \subseteq$ Aut $H_{*}(M)$ consists of all $\varphi$ such that $\varphi=k_{*}$ for some diffeomorphism $k$. Let $C$ consist of all $\varphi$ such that $\varphi=k_{*}$ for some homotopy self-equivalence $k$, so that $A / B \subseteq C / B$. According to 1.5 below, $B$ and $C$ are equal if $M$ is a product of spheres, and hence in this case $A$ and $B$ are also equal. Thus if $M$ is a product of spheres, we have $I(M)=I_{0}(M)$. 
1.3. If $f$ is any homotopy self-equivalence of a product of spheres, then $f$ induces automorphisms of the subgroups of spherical homology classes. Hence one obtains a collection of square matrices with integral entries and determinants \pm 1 , one matrix for each factor dimension appearing. These matrices are further restricted as follows:

LEMMA. Let $A$ represent the induced automorphism of $k$-dimensional spherical homology classes with respect to the standard basis.

(i) If $k$ is even, $A$ is a permutation matrix up to the signs of the factors.

(ii) If $k$ is odd and $k \neq 1,3,7$, then $A$ reduced $\bmod 2$ is a permutation matrix.

Conversely, any square matrix $A$ satisfying the above restrictions is induced by $a$ diffeomorphism of a product of $k$-spheres.

Proof. Case 1. $k$ even. It will be more convenient to consider the dual automorphism of spherical cohomology classes, whose matrix is the transpose of $A$. If one considers the standard basis of spherical cohomology classes, then the fact that cup products are preserved implies that the image of a standard basis class (whose cup square vanishes) must be a multiple of some basis element. An easy algebraic argument now implies that the transpose of $A$ must be a permutation matrix up to the signs of the entries; clearly $A$ must also be of the same type.

Conversely, it is immediate that any matrix satisfying (i) is induced by a selfequivalence of $k$-spheres.

Case 2. $k$ odd, $k \neq 1,3,7$. As above we look at the induced cohomology automorphism. In this case all $k$-dimensional cup squares vanish; hence, in analogy with the formulation of the Arf invariant, we are forced to consider higher order operations. Since $k \neq 1,3,7$, there is a second or third order cohomology operation

$$
\varphi: \operatorname{Sub} H^{k}\left(X ; Z_{2}\right) \rightarrow \text { Quot } H^{2 k}\left(X ; Z_{2}\right)
$$

which detects the Whitehead product; for further discussion see [5]. Since all the cohomology groups of a product of spheres have bases of cospherical classes, it is immediate that $\varphi$ is everywhere defined and has no indeterminacy. Using the naturality of $\varphi$ we may argue much as before that $A$ reduced mod 2 is a permutation matrix.

The converse in this case is somewhat less trivial to show; we introduce some notation. Two square matrices $A, B$ are permutation conjugate if there is a permutation matrix $P$ such that $B=P^{-1} A P$. The matrix $C$ is an extended $2 \times 2$ matrix if it is obtained by placing a $2 \times 2$ matrix in the upper left corner, ones down the rest of the diagonal, and zeros elsewhere.

Sublemma. Let $A$ be an invertible matrix over the integers which is the identity when reduced mod 2. Then there exist unimodular matrices $C_{r}, D_{s}$ over the integers such that 
(i) Each $C_{r}, D_{s}$ is the identity $\bmod 2$.

(ii) Each matrix is permutation conjugate to an extended $2 \times 2$ matrix of determinant \pm 1 .

(iii) There is the relation

$$
C_{1} \cdots C_{m} A D_{1} \cdots D_{m}=I
$$

This result may be proved by reviewing the proof of the elementary divisor theorem [10, pp. 83-84] and noticing that at each step one may choose $C_{r}, D_{s}$ as claimed.

We now prove the converse of Case 2. If $B$ satisfies the hypothesis above, by means of the Sublemma express $B$ as a product $\prod B_{i}$ of matrices $B_{i}$ which satisfy (i) and (ii). By a result of Wall [31, Lemma 5], there exist self-diffeomorphisms $f_{i}$ of a product of $k$-spheres which induce the matrices $B_{i}$ in homology. Thus the product of the $f_{i}$ induces $B$ in homology.

Case 3. $k=1,3,7$. We claim that any invertible matrix over the integers is induced by some diffeomorphism. This is shown for $2 \times 2$ matrices in [17, 1.2]. More generally, any invertible $n \times n$ matrix may be written as a product of matrices which are permutation conjugate to extensions of invertible $2 \times 2$ matrices. As in Case 2, construction of the required diffeomorphism is immediate.

1.4. We now consider self-equivalences of a product of spheres such that the induced automorphism of spherical homology classes is the identity. Let $P$ be a fixed product of the spheres $S_{1}, \ldots, S_{k}$ where the dimension of $S_{i}$ is $d(i)$.

Let $G_{q+1}$ be the topological monoid of orientation-preserving homotopy selfequivalences of $S^{q}$.

LEMMA. Let $h$ be a homotopy self-equivalence of $P$ which induces the identity automorphism of spherical homology classes. Then $h$ is homotopic to a product $\prod h_{i}$, where $h_{i}$ is a fiber homotopy equivalence of $P$ when considered to be the trivial $S_{i}$ bundle over the remaining factors.

Proof. Let $Q_{i}$ be the product of the first $(i-1)$ factors, let $R_{i}$ be the product of the last $(k-i)$ factors, and let $P_{i}=Q_{i} \times R_{i}$; by definition $P=P_{i} \times S_{i}$. The projections of $P$ onto $Q_{i}$ and $R_{i}$ are denoted by $q_{i}$ and $r_{i}$ respectively, and $\pi_{i}: P \rightarrow S_{i}$ is the ordinary projection. We first observe that it suffices to prove the result for selfequivalences $f: S_{1} \times P_{1} \rightarrow S_{1} \times P_{1}$ satisfying $\pi_{1} f \simeq \pi_{1}$. For $\pi_{1} f: S_{1} \times P_{1} \rightarrow S_{1}$ is adjoint to some $f^{\prime}: P_{1} \rightarrow G_{d(1)+1}$ by homological considerations, and if $g$ is a homotopy inverse to $f^{\prime}$ with adjoint self-equivalence $g_{\#}$, then $f_{1}=f g_{\#}$ has the property $\pi_{1} f_{1} \simeq \pi_{1}$. Since $f_{1}$ factors up to homotopy, so does $f \simeq f_{1} f_{\# \text {. }}^{\prime}$.

Assume by induction that it suffices to prove the Lemma for self-equivalences $f$ satisfying $q_{i} f \simeq q_{i}$. (For convenience assume $k \geqq 3$, since a proof for $k=2$ may be obtained by skipping this paragraph; alternatively, this case is proved in [17, $\S \S 2-3]$.) We shall show that it suffices to prove the Lemma for $f$ satisfying $q_{i+1} f$ 
$=q_{i+1}$; i.e., $q_{i} f=q_{i}$ and $\pi_{i} f=\pi_{i}$. Again take $f^{\prime}: P_{i} \rightarrow G_{d(i)+1}$ to be adjoint to $\pi_{i} f$, and assume $q_{i} f=q_{i}$. If $g$ is inverse to $f^{\prime}$, consider $h=f g_{\#}$; as before, we have $\pi_{i} h \simeq \pi_{i}$, but we also know that

$$
q_{i} h=q_{i} g_{\#} \simeq q_{i} g_{\#}=q_{i}
$$

by our hypotheses on $f$ and the construction of $g_{\#}$. This finishes the inductive step and thus implies that it suffices to show the Lemma when $q_{k} f \simeq q_{k}$; without loss of generality, they may be assumed equal.

But $Q_{k}=P_{k}$, and the fact that $f$ commutes with projection onto $P_{k}$ implies that $f=g_{\#}$ for some suitable $g: P_{k} \rightarrow G_{d(k)+1}$. This proves the Lemma.

REMARK. If $h$ induces the identity in homology, then the $h_{i}$ may be chosen likewise.

1.5. Corollary. Let $h$ be a homotopy self-equivalence of $P$. Then there is a diffeomorphism $g$ of $P$ which induces the same automorphism of homology as $h$.

Proof. By 1.3 we can find a diffeomorphism which induces the same automorphisms of spherical homology classes as $h$, so without loss of generality we may assume that $h$ induces the identity on spherical homology classes. Thus by 1.4 we are reduced to considering the induced homology automorphisms of fiber homotopy equivalences represented by homotopy classes in the groups $\left[P_{i}, G_{d(i)+1}\right]$. The latter group is generated by various homotopy groups of $G_{d(i)+1}$, and the identity automorphism of homology is always induced unless there is a factor of the form $\pi_{d(i)}\left(G_{d(i)+1}\right)$. Furthermore, it is well known (compare [1], [13]) that each element of this homotopy group may be written as the sum of an element inducing the identity in homology with an element in the image of $\pi_{d(i)}\left(S O_{d(i)+1}\right)$. In other words, every homology automorphism represented by an element of $\pi_{d(i)}\left(G_{d(i)+1}\right)$ may also be represented by an element of $\pi_{d(i)}\left(S O_{d(i)+1}\right)$ and consequently by a diffeomorphism.

1.6. Proof of Theorem A. Suppose $h: P \# \Sigma \rightarrow P$ is an orientation-preserving diffeomorphism. Then by 1.5 there is a diffeomorphism $f$ of $P$ such that $f h$ and $i$ induce the same mapping in homology (and hence in fundamental groups). Consequently, $\Sigma \in I_{0}(M)$ (see 1.1) and by 1.2 we have that $\Sigma$ is the ordinary sphere.

1.7. We give an easy application of Theorem A which was motivated by the case where $M$ is a product of two spheres. Let $D_{n}$ be the group of diffeomorphisms of the $n$-disk which are the identity near the boundary. If Diff $M$ is the diffeomorphism group of the closed $n$-manifold $M(n \geqq 6)$, then by means of a coordinate chart one may form an inclusion mapping $\sigma: D_{n} \rightarrow$ Diff $M$. By the Cerf-Palais disk theorem the homotopy class of $\sigma$ is independent of the choice of chart. Hence there is a well-defined mapping

$$
\sigma_{*}: \Gamma_{n+1}=\pi_{0}\left(D_{n}\right) \rightarrow \pi_{0}(\operatorname{Diff} M) .
$$


Proposition. If $M$ is a product of ordinary spheres, then $\sigma_{*}$ is a monomorphism. In fact, if $\tilde{\pi}_{0}(\mathrm{Diff} M)$ is the group of concordance classes of diffeomorphisms of $M$ [32], then the mapping

$$
\Gamma_{n+1} \rightarrow \pi_{0}(\operatorname{Diff} M) \rightarrow \tilde{\pi}_{0}(\operatorname{Diff} M)
$$

is a monomorphism.

The proof of this fact when $M$ is a product of two spheres $\left(M=S^{p} \times S^{q}\right)$ is contained in the results of Levine [17], Sato ([24], [25]), and Turner [30] on $\pi_{0}\left(\right.$ Diff $\left.S^{p} \times S^{q}\right)$.

Proof. If $\beta \in \Gamma_{n+1}$ goes to zero under the above map, then it follows that the mapping torus of $\sigma_{*} \beta$ is diffeomorphic to the mapping torus of the identity. Since these mapping tori are $M \times S^{1} \# S_{\beta}^{n+1}$ and $M \times S^{1}$ respectively, the result follows since $I\left(M \times S^{1}\right)=0$.

\section{Generalizations.}

2.1. The methods of $\S 1$ are of such a straightforward nature that one is immediately confronted with the problem of generalizing them. Instead of considering a product of ordinary spheres, it is possible to consider the product $\Sigma^{n} \times P^{k}$ of a homotopy sphere $\Sigma^{n}$ with a product $P^{k}$ of ordinary spheres such that $\Sigma^{n} \times P^{k}$ embeds in $R^{n+k+1}$; this always happens if $k \geqq \max (n-3,7-n)$, since $\Sigma^{n}$ then embeds in $R^{n+k+1}$ with trivial normal bundle [8]. In the metastable range $k \geqq(n+1) / 2$ this is still the case for a wide variety of homotopy spheres; separate results along these lines are due to Novikov [22] and Antonelli [2]. (The latter may be generalized using results of Mahowald [18].) In any such example we should assume that the highest dimensional factor of $P^{k}$ has dimension $\leqq n-4$ (provided $n \geqq 5$ ), since otherwise $\Sigma^{n} \times P^{k}$ is diffeomorphic to $S^{n} \times P^{k}$ and hence any generalization is empty. Proposition 1.2 will still hold for $\Sigma^{n} \times P^{k}$, and hence to show that $I\left(\Sigma^{n} \times P^{k}\right)=0$ it suffices to look again at the possible automorphisms of homology.

By the reasoning of 1.5 , we may assume that any diffeomorphism

$$
h: \Sigma^{n} \times P^{k} \# U^{n+k} \rightarrow \Sigma^{n} \times P^{k}
$$

induces the same map in homology as $g_{\#} i$, where $g$ represents an element of $\left[P^{k}, G_{n+1}\right]$. A more detailed analysis of the Mayer-Vietoris sequence than that of 1.2 is now necessary. A basis for $H_{q}(\Sigma \times P)$ may be constructed consisting of monomials in the fundamental classes of $\Sigma$ and the factors of $P$ (multiplication is by the external homology cross product). A straightforward computation shows that the matrix of $h_{*}$ is unitriangular with respect to such a basis; for cup product considerations imply that a monomial $\mu$ with $r$ factors is sent by $h_{*}$ into $\mu+\tau$, where $\tau$ is a linear combination of monomials with less than $r$ factors. A direct examination of the Mayer-Vietoris sequence as in 1.2 now shows that the manifold $X$ with $\partial X=U$ (same notation as in 1.2) is again acyclic; this follows readily from the unitriangularity of the matrix of $h_{*}$. Since all 1-dimensional homology classes 
are spherical and the Hurewicz map is an isomorphism on $\pi_{1}(\Sigma \times P), X$ is again simply connected; this concludes the proof that $X$ is contractible and $U=S^{n+k}$.

We have remarked above that the stated generalization may well be empty. One well-known generalization of a nontrivial nature is the case $P^{k}=T^{k}$, which suggests that the almost diffeomorphism class of $\Sigma^{n} \times P^{k}$ should depend only on the dimensions of the factors of $P^{k}$; this will be shown in subsection 2.3.

2.2. In this subsection we recall how smoothing theory [15] and the SullivanWall reformulation of Browder-Novikov theory ([21], [29], [33]) give related methods for determining whether two smoothings of a manifold $M$ are diffeomorphic. We include general statements of these fairly standard principles and formulas in order to explain at least part of the formalism involved in the routine computational proofs of some assertions which appear later.

First consider smoothing theory. Assume $M$ is smoothable and has a preferred basic smoothing $\left(M_{0}, t_{0}\right)$. The group $\mathscr{P}(M)$ of concordance classes of PL homeomorphisms of $M$ acts on the right of the set $[M, P D / O]$ of concordance classes of combinatorial smoothings of $M$ as follows: If $(N, t)$ is a smoothing of $M(t: M \rightarrow N$ is a smooth triangulation) representing the concordance class $\alpha$ and $h$ is a PL homeomorphism of $M$ representing $\varphi \in \mathscr{P}(M)$, then the concordance class of $(N, t h)$ is defined to be the element $\alpha \cdot \varphi$; this class does not depend on the choice of representatives for $\alpha$ and $\varphi$. Then two concordance classes of smoothings are diffeomorphic if and only if they are in the same orbit under the action of $\mathscr{P}(M)$. It is generally difficult to compute this action explicitly. However, the following relationship is useful. Suppose $\alpha_{0}$ is the basic smoothing of $M$; i.e., under the isomorphism between the set of concordance classes of smoothings of $M$ and the group $[M, P D / O]$ (compare $[15, \S 6]), \alpha_{0}$ corresponds to the identity. Then the action of $\varphi \in \mathscr{P}(M)$ on an arbitrary smoothing $\alpha$ is given by the formula

$$
\alpha \cdot \varphi=\alpha_{0} \cdot \varphi+h^{*} \alpha,
$$

where $h^{*} \alpha$ represents the homotopy composition $\alpha[h]$.

There are several obstacles to the effective computability of the above action, the most immediate of which is the computation of the group $\mathscr{P}(M)$; to the best of our knowledge, the only explicit results along this line are for complex projective spaces [29], products of two spheres ([11], [17]) and tori ([9], [34]). It is therefore reasonable to work within a framework depending upon the more accessible group $\mathscr{E}(M)$ of homotopy self-equivalences.

We do this by means of surgery theory. The group $\mathscr{E}(M)$ acts on the left of the set $h S(M)$ of homotopy smoothings by the formula $\varphi \cdot(X, h)=(X, \varphi h)$, where $h: X \rightarrow M$ is a homotopy smoothing. As before, two homotopy smoothings determine diffeomorphic manifolds if and only if they are in the same orbit of $\mathscr{E}(M)$. Of course there is a natural map basically defined by taking the combinatorial smoothing $(N, t)$ to the homotopy smoothing $\left(N, t^{-1}\right)$. Under this map, the action of an element of $\mathscr{P}(M)$ goes over to the action of the inverse of its 
representative in $\mathscr{E}(M)$. In analogy with formula (i), it is possible to deduce something about the action of $\mathscr{E}(M)$ on an arbitrary element of $h S(M)$ from its effect on the obvious basepoint $(M$, id). Let $q: h S(M) \rightarrow[M, G / O]$ be the map which sends each homotopy smoothing to its normal invariant. Then for any $\alpha \in h S(M)$, the normal invariant of $\varphi \cdot \alpha$ is given by the following formula:

$$
q(\varphi \cdot \alpha)=\left(\varphi^{-1}\right)^{*} q(\alpha)+q(\varphi \cdot O) .
$$

We give an example of the action of $\mathscr{E}(M)$ on $h S(M)$ which will be used later; it is essentially due to Novikov [21, §11]. Let $M=S^{n} \times S^{k}$, and let $\varphi \in \mathscr{E}\left(S^{n} \times S^{k}\right)$ be determined by the class $\theta \in \pi_{n}\left(G_{k+1}\right)$. If $s: G_{k+1} \rightarrow G / O$ is the obvious mapping, then $q(\varphi \cdot O)=-s_{*} \theta$. (The sign discrepancy arises because the results of [21] have been reformulated here.)

Finally we say something about combinatorial smoothings which give the trivial homotopy smoothing. Such a smoothing is of the form $\alpha_{0} \cdot \varphi$, where $\varphi$ is a PL selfequivalence of $M$ which is homotopic to the identity. The study of such maps involves the $\pi_{1}$ portion of the long exact sequence of surgery theory [33], and it is not hard to see that the above set of smoothings may be given as follows. Let $S:[M, \Omega(G / P L)] \rightarrow L_{n+1}(M)$ be the map which takes a 1-simplex of normal mappings to its surgery obstruction, and let $\partial: \Omega(G / P L) \rightarrow P D / O$ be the canonical map in the extended fibration sequence of

$$
P D / O \rightarrow G / O \rightarrow G / P L
$$

Then the concordance classes of smoothings which are homotopy equivalent to the identity are the elements of the set $\partial_{*}(\operatorname{Kernel} S)$.

2.3. Proposition. Let $\alpha, \beta \in \Gamma_{n}$, and let $S_{1}$ be a factor of $P$ having maximal dimension. Then $S_{\beta}^{n} \times P$ and $S_{\alpha}^{n} \times P$ are almost diffeomorphic if and only if $S_{\beta}^{n} \times S_{1}$ and $S_{\alpha}^{n} \times S_{1}$ are, in which case they are diffeomorphic.

In particular, we see that there are many nontrivial examples of products $S_{\beta}^{n} \times P^{k}$ where $k \geqq n-3$ and $P^{k}$ is simply connected.

We assume $n$ is greater than the dimension of any factor of $P$.

Proof. The "if" direction is immediate, since the two products $S_{\beta}^{n} \times S_{1}$ and $S_{\alpha}^{n} \times S_{1}$ are almost diffeomorphic if and only if they are diffeomorphic $[26,1.5]$.

Before proving the "only if" direction, we make the trivial observation that in order to classify the homotopy smoothings of a product $Q$ of ordinary spheres up to a diffeomorphism which is the identity on spherical homology classes it is necessary to look at the orbits of the subgroup $\mathscr{E}_{1}(M) \subseteq \mathscr{E}(M)$ of homotopy selfequivalences which induce the identity on spherical homology classes. This is more convenient for our purposes since in 1.4 we obtained a set of generators for $\mathscr{E}_{1}(M)$. Furthermore, it is clear (compare 1.5) that if the manifolds $S_{\alpha}^{n} \times P$ and $S_{\beta}^{n} \times P$ are almost diffeomorphic, then there is an almost diffeomorphism which induces the identity in spherical homology. 
It will simplify matters to factor an element of $\mathscr{E}_{1}\left(S^{n} \times P^{k}\right)$ in a special manner. Let $S_{1}, \ldots, S_{l}$ be the factors of $P^{k}$; then any element of $\mathscr{E}_{1}\left(S^{n} \times P^{k}\right)$ may be expressed as a product

$$
k_{1} h_{1} \cdots k_{l} h_{l} h^{\prime}
$$

where the map $h^{\prime}$ is a fiber homotopy equivalence with fiber $S^{n}$ and base $P^{k}$, the map $h_{i}$ is a fiber homotopy equivalence with fiber $S_{i}$ and base $S^{n} \times P_{i}$ (see 1.4 for definition of $P_{i}$ ) which is the identity on $S^{n}$, and $k_{i}=k_{i}^{\prime} \times 1$, where $k_{i}^{\prime}$ is a fiber homotopy equivalence with fiber $S_{i}$ and base $S^{n}$. The proof that such factorizations exist is a straightforward formal exercise based on 1.4.

Let $j: P D / O \rightarrow G / O$ be the usual inclusion. Then the normal invariant of the smoothing $S_{\alpha}^{n} \times P$ is given by

$$
j_{*} \alpha \in \pi_{n}(G / O) \subseteq\left[S^{n} \times P, G / O\right]
$$

We wish to examine the effect that each fiber homotopy equivalence in the above factorization has on the $\pi_{n}(G / O)$ component of $\left[S^{n} \times P, G / O\right]$. It is not difficult to show that $h^{\prime}$ and the $h_{i}$ do not affect this factor at all. Let $\theta_{i} \in \pi_{n}\left(G_{d(i)+1}\right)$ be the homotopy class determined by $k_{i}$, let $s_{i}: G_{d(i)+1} \rightarrow G / O$ be the obvious map, and let $e_{i}: G_{d(i)+1} \rightarrow S_{i}$ be the evaluation map. Then the net effect of the $k_{i}$ on the $\pi_{n}$ component is to add terms of the form $-s_{i *} \theta_{i}$ and $\psi_{i *}\left(e_{i *} \theta_{i}\right)$, where $\psi_{i} \in \pi_{d(i)}\left(G_{d(i)+1}\right)$. In particular, if $S_{\beta}^{n} \times P$ and $S_{\alpha}^{n} \times P$ are diffeomorphic, then $j_{*} \beta \equiv j_{*} \alpha \bmod$ image $s_{1 *}$. (Recall $d(1) \geqq d(i)$, all $i$.) But by the results of Levine ([16], [23]), this implies that $S_{\beta-\alpha}^{n}$ embeds in $R^{d(i)+1}$ with trivial normal bundle, and hence by $[26,1.8]$ and $S_{\alpha}^{n} \times S_{1}$ are diffeomorphic.

2.4. We make an observation on the inertia group of $S_{\beta}^{n} \times P^{k}$ when $k \leqq n-4$. In this case all fiber homotopy equivalences in $\left[P^{k}, G_{n+1}\right]$ induce the identity in homology, and hence $S_{\beta}^{n} \times P^{k} \# S_{\gamma}^{n+k}$ and $S_{\beta}^{n} \times P^{k}$ are diffeomorphic if and only if $S_{\beta}^{n} \times S_{1} S_{\beta}^{n} \times S_{1}$ some diffeomorphism induces the identity in homology.

\section{Manifolds homeomorphic to $S^{n} \times T^{k}$.}

3.1. In this section we shall express the inertia group of $S_{\beta}^{n} \times T^{k}$ in homotopytheoretic terms. Actually, we shall classify up to homology-preserving diffeomorphism all smooth manifolds homeomorphic to $S^{n} \times T^{k}$, where $n \geqq 4$ and $1 \leqq k \leqq n-4$. This result is a fairly straightforward analog of the classification theorem for $S^{n} \times S^{k}$ (compare [26, p. 187]).

We first introduce some notation. The group $\left[T^{k}, S O_{n+1}\right]$ acts on $\left[S^{n} \times T^{k}, Y\right]$ (any $Y$ ) as follows: Take an $S^{n}$ bundle automorphism $f_{\#}$ of $T^{k} \times S^{n}$ induced by a

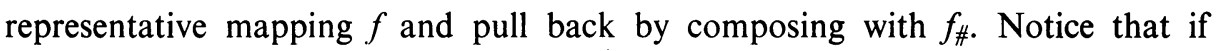
$W=S^{n} \vee\left(S^{n} \wedge T^{k}\right)$ and $q: S^{n} \times T^{k} \rightarrow S^{n} \times T^{k} / T^{k} \simeq W$ is the canonical projection, then this action of $\left[T^{k}, S O_{n+1}\right]$ maps the image of the induced mapping

$$
q^{*}:[W, Y] \rightarrow\left[S^{n} \times T^{k}, Y\right]
$$

into itself. Furthermore, if $Y$ is an iterated loop space, we shall give a computation of this action in 3.3 and the Appendix. 
3.2. THEOREM B. Under the above assumptions, any manifold $M$ homeomorphic to $S^{n} \times T^{k}$ is diffeomorphic to a smoothing $\left(M_{\alpha}, t_{\alpha}\right)$ whose concordance class is in the image of $q^{*}:[W, P D / O] \rightarrow\left[S^{n} \times T^{k}, P D / O\right]$. Two such smoothings $q^{*} \alpha, q^{*} \beta$ are diffeomorphic by a map $h: M_{\alpha} \rightarrow M_{\beta}$ such that $h t_{\alpha}$ and $t_{\beta}$ induce the same map in homology if and only if $\alpha$ and $\beta$ are in the same orbit of $\left[T^{k}, S O_{n+1}\right]$.

Proof. First notice that any smoothing of $S^{n} \times T^{k}$ whose concordance class lies in the image of $\left[T^{k}, P D / O\right]$ is in the orbit of the basic smoothing under a selfequivalence of $S^{n} \times T^{k}$ given by an element of [ $T^{k}, P L_{n}$ ], and consequently by 2.2(i) it is possible to find a smoothing diffeomorphic to the given one whose concordance class restricts trivially to $\left[T^{k}, P D / O\right]$. In other words, it is an image of $q^{*}$; likewise for $T o p / O, T_{o p}$.

If the concordance classes $\alpha, \beta$ determine diffeomorphic smoothings as stated in the theorem, then as homotopy smoothings they are in the same orbit of $\mathscr{E}_{1}\left(S^{n} \times T^{k}\right)$. But by 1.4 this group is merely $\left[T^{k}, G_{n+1}\right]$, and the condition that the smoothings have no $T^{k}$ component implies that the group element is in the image of $\left[T^{k}, S O_{n+1}\right]$. Since the elements of the latter group act diffeomorphically on $S^{n} \times T^{k}$, the action on normal invariants in [W, $G / O$ ] is merely given by composition; a similar statement holds for $[W, P D / O]$. Hence by the general theory we know that $\alpha, \beta$ are diffeomorphic as desired if they are in the same orbit of $\left[T^{k}, S O_{n+1}\right]$ on $[W, P D / O]$. Furthermore, if a diffeomorphism exists with the desired properties, then the homotopy smoothings in question are in the same orbit of $\left[T^{k}, S O_{n+1}\right]$ on $[W, G / O]$. This means that for some group element $g, g \alpha$ and $\beta$ are equivalent homotopy smoothings.

It is therefore sufficient to show that two smoothings of the special type are concordant if they are homotopy equivalent. We must accordingly determine the intersection of the set $\partial_{*}($ Kernel $S)$ of homotopically trivial smoothings described at the end of 2.2 with the image of $q^{*}$. But by the Shaneson-Wall computation of $L_{n+k+1}\left(S^{n} \times T^{k}\right)$ ([28], [33]), the mapping $S$ corresponds to the homomorphism

$$
\left[S^{n} \times T^{k}, \Omega(G / P L)\right] \stackrel{r}{\longrightarrow}[W, \Omega(G / P L)] \longrightarrow[W, \Omega(G / T o p)],
$$

where $r$ is a one-sided inverse to $q^{*}$. Hence the kernel of $S$ consists of $\left[T^{k}, \Omega(G / P L)\right]$, and its image in $\left[T^{k}, P D / O\right]$ clearly intersects that of $[W, P D / O]$ trivially. This concludes the proof that $g \alpha$ and $\beta$ are identical.

3.3. We shall comment on the computation of the group action on $\left[S^{n} \times T^{k}, P D / O\right]$. Recall that if $P$ is a product of spheres and $H$ is a homotopy associative and commutative $H$-space, then the group $[P, H]$ is a direct sum $\sum \pi_{|A|}(H)$, where $A$ runs over all nonempty subsets of the set of factors of $P$ and $|A|$ is the sum of the dimensions of the factors belonging to $A$. Thus $\left[T^{k}, S O_{n+1}\right]$ and $[W, P D / O]$ may be respectively expanded as follows:

$$
\begin{aligned}
{\left[T^{k}, S O_{n+1}\right] } & =\sum \pi_{|A|}\left(S O_{n+1}\right), \\
{[W, P D / O] } & =\pi_{n}(P D / O) \oplus \sum \pi_{|B|+n}(P D / O) .
\end{aligned}
$$


It certainly suffices to compute the $g_{A} \alpha_{B}$ (and $g_{A} \alpha_{n}$ ) separately. There are several distinct cases; the derivations are given in the Appendix.

(i) $g_{A} \alpha_{n}=\alpha_{n}+\left(\alpha_{n} J\left(g_{A}\right)\right)_{A}$.

(ii) If $A \cap B=\varnothing$, then $g_{A} \alpha_{B}=\left(\alpha_{B} J\left(g_{A}\right)\right)_{A \cup B}$.

(iii) If $A \cap B \neq \varnothing$, then $g_{A} \alpha_{B}=\alpha_{B}$.

3.4. The homology automorphisms of $S^{n} \times T^{k}$ are given by the group of diffeomorphisms $Z_{2} \times \mathrm{GL}(k, Z)$. Thus by 2.2(ii) there are standard smoothings which are diffeomorphic by an arbitrary homology automorphism, and one smoothing may be computed from the other by means of homotopy composition. As an "application" of the criteria given here and above, we have tabulated the number of differentiably inequivalent products in some simple cases and listed the results below.

\begin{tabular}{|l|c|c|r|r|}
\hline \multicolumn{1}{|c|}{ Manifold } & $S^{6} \times T^{2}$ & $S^{7} \times T^{2}$ & $S^{10} \times T^{2}$ & $S^{10} \times T^{3}$ \\
\hline $\begin{array}{l}\text { Oriented or } \\
\text { unoriented } \\
\text { almost } \\
\text { diffeomorphism } \\
\text { classes }\end{array}$ & 15 & 30 & 1988 & 1988 \\
\hline $\begin{array}{l}\text { Oriented } \\
\text { diffeomorphism } \\
\text { classes }\end{array}$ & 30 & 180 & 1988 & 3976 \\
\hline $\begin{array}{l}\text { Unoriented } \\
\text { diffeomorphism } \\
\text { classes }\end{array}$ & 30 & 180 & 1988 & 2982 \\
\hline $\begin{array}{l}\text { Concordance } \\
\text { classes of } \\
\text { smoothings }\end{array}$ & 1568 & 896 & $5,904,384$ & $17,561,445,984$ \\
\hline
\end{tabular}

TABLE. Number of differentiably inequivalent smoothings of some topological manifolds under various relations

3.5. Corollary. If $n \geqq 5$ and $k \leqq n-4$, then $I\left(S_{\beta}^{n} \times T^{k}\right)=I\left(S_{\beta}^{n} \times S^{k}\right)$.

Proof. Let

$$
\beta_{0}=\beta \in \pi_{n}(P D / O) \text { and } \beta_{1}=(\beta, \gamma) \in \pi_{n}(P D / O) \oplus \pi_{n+k}(P D / O) .
$$

By 2.4 we may assume $\beta_{0}$ and $\beta_{1}$ are diffeomorphic by a map which induces the identity in homology. A direct examination of 3.3 now shows that $g\left(\beta_{0}\right)=\beta_{1}$ if and only if $g \in \pi_{n}\left(S O_{n+1}\right)$ and $\gamma=\beta J(g)$. But these elements are precisely those of the inertia group of $S_{\beta}^{n} \times S^{k}[26,2.5]$.

3.6. REMARK. We have not considered products involving exotic 3- and 4dimensional homotopy spheres, the reason being that if $\Sigma^{n}$ is a homotopy sphere and $n=3$ or 4 , then $\Sigma^{n} \times P^{k}=S^{n} \times P^{k}$ unless $n=3$ and $P^{k}$ is the $k$-dimensional torus [7]. In the latter case, by surgery theory $\Sigma^{3} \times T^{k} \neq S^{3} \times T^{k}(k \geqq 2)$ if and only if $\Sigma^{3}$ 
bounds a framed 4-manifold of index 8. On the other hand, Shaneson has constructed a manifold $M^{5}$ which is diffeomorphic to $\Sigma^{3} \times T^{2}$ if such a $\Sigma^{3}$ exists [28, $\S 7$. By the remarks made in 2.1 , we know $I\left(\Sigma^{3} \times T^{k}\right)=0$ for all $\Sigma^{3}$, and hence one would naturally guess that $I\left(M^{5} \times T^{k}\right)=0$. This may be shown directly by means of the techniques used in this paper.

\section{The inertia group of $\Sigma^{n} \times P^{k}$.}

4.1. Of course, if $P^{k} \neq T^{k}$ then the structure of $\mathscr{E}_{1}\left(S^{n} \times P^{k}\right)$ is considerably more complicated. In particular, if $f$ represents an element of this group, then $\pi_{P} f \mid P^{k}$ might not be homotopic to the identity. We shall first dispose of the special case where this mapping is the identity; the assumption $k \leqq n-4$ will hold throughout this subsection.

LEMMA. There is an orientation-preserving diffeomorphism $h$ from $S_{\beta}^{n} \times P^{k} \# S_{\gamma}^{n+k}$ to $S_{\beta}^{n} \times P^{k}$ such that $\pi_{P} h \mid P$ is the identity if and only if $\gamma=\beta J(\alpha)$ for some $\alpha \in \pi_{k}\left(S O_{n}\right)$.

REMARK. The condition $\pi_{P} h \mid P=\mathrm{id}$ implies that $h$ induces the identity in homology.

Proof. If such a diffeomorphism exists, then there is an element $\varphi \in \mathscr{E}\left(S^{n} \times P^{k}\right)$ such that $\pi_{P} \varphi \mid P=1_{P}$ and $\varphi \cdot(\beta, 0)=(\beta, \gamma)$. Much as in 2.3 , we factor $\varphi$ into a product of the form

$$
f k_{1} h_{1} \cdots k_{l} h_{l}
$$

where the map $f$ is a fiber homotopy equivalence with fiber $S^{n}$ and base $P^{k}$, the map $k_{i}=k_{i}^{\prime} \times 1$, where $k_{i}^{\prime}$ is a fiber homotopy equivalence with fiber $S_{i}$ and base $S^{n}$, and the map $h_{i}$ is a fiber homotopy equivalence with fiber $S_{i}$ and base $S^{n} \times P_{i}$ which is the identity on $S^{n} \vee P_{i}$ (i.e., it comes from an element of $\left.\left[S^{n} \wedge P_{i}, G_{d(i)+1}\right]\right)$.

As in 2.3, we compute the effect of each factor in turn. In particular, at each point in the computation, except perhaps the last, any homotopy smoothing whose normal invariant in $\left[S^{n} \times P^{k}, G / O\right]$ vanishes on the $\left[P^{k}, G / O\right]$ component is taken to another one with the same property. This also happens at the last step if and only if $f$ is in the image of $\left[P^{k}, S O_{n+1}\right]$. Hence a diffeomorphism as required exists if and only if $f^{-1}(\beta, \gamma)=\left(\prod k_{i} h_{i}\right) \cdot(\beta, 0)$. We basically computed the action of $f^{-1}$ in 3.3. The action on the right-hand side is basically that of adding elements in the image of $\pi_{n+|A|}\left(G_{d(A)+1}\right)$, where $A$ is a proper (possibly empty) subset of $\{1, \ldots, l\}$ and $d(A)$ is the dimension of the largest factor sphere of $P^{k}$ not contained in $A$. In particular, the $\pi_{n+k}(G / O)$ component is zero.

Notice that if $f$ represents $-\alpha \in \pi_{k}\left(S O_{n+1}\right) \subseteq\left[P^{k}, S O_{n+1}\right]$, then $f(\beta, 0)=(\beta, \beta J(\alpha))$ and hence the "if" portion of the Lemma follows.

By the reasoning of 2.3 , this implies that the component $\gamma_{A}$ of $f^{-1}(\beta, \gamma)$ in $\pi_{n+|A|}(G / O)$ is in the image of $\pi_{n+|A|}\left(G_{d(A)+1}\right)$ provided $A$ is a nonempty proper subset. Since the natural inclusion

$$
G_{s} /\left(P L_{s}^{\sim}\right) \rightarrow G / P L
$$


is a homotopy equivalence for $s \geqq 3$ ([16], [23]), we know that $\gamma_{A}$ is in the image of $\pi_{n+|A|}\left(P L_{\tilde{d}(A)+1}\right)$; i.e., the homotopy sphere it represents embeds in Euclidean space of codimension $d(A)+1$ with trivial normal bundle [16]. By the computation of 3.3, we see that the $\pi_{n+k}(G / O)$ component of $f^{-1}(\beta, \gamma)$ is the sum of $j_{*}(\gamma-\beta J(\alpha))$ (some $\alpha \in \pi_{k}\left(S O_{n}\right)$ ) with terms of the form $\gamma_{A} J\left(\xi_{1}\right) \cdots J\left(\xi_{r}\right)$, where the $\xi_{i}$ are in homotopy groups of the orthogonal group and in particular $\xi_{1} \in \pi_{q}\left(S O_{p}\right)$ for some $p \geqq q+2 \geqq d(A)$. However, by the results of $[26,1.8,2.6]$ we know that $\gamma_{A} J\left(\xi_{1}\right)=0$. Thus the entire sum is $j_{*}(\gamma-\beta J(\alpha))$, which by the previous computation of $\left(\prod k_{i} h_{i}\right) \cdot(\beta, 0)$ vanishes in $\pi_{n+k}(G / O)$. In other words, $\gamma-\beta J(\alpha) \in \partial P_{n+k+1}$.

This difference actually vanishes. If the normal invariant of $\varphi_{1}=f^{-1} \varphi$ is computed in the homotopy triangulation theory, it will vanish basically because the images of the $\gamma_{A}$ in $\pi_{n+|A|}(G / P L)$ are zero by the previous paragraph. Hence by surgery theory $\varphi_{1}$ is homotopic to a PL homeomorphism $\psi_{1}$, and we have that $f(\beta, \gamma)$ and $\beta \cdot \psi_{1}$ are equivalent as homotopy smoothings. By the special homotopy-theoretic nature of $\varphi_{1}$ as displayed above, we may write

$$
\beta \cdot \psi_{1}=\beta+0 \cdot \psi_{1} \text {. }
$$

Furthermore, $0 \cdot \psi_{1}$ may be chosen to be a sum of terms $\gamma_{A}^{\prime}$ where $A$ runs over all proper subsets of $\{1, \ldots, l\}$ and $j_{*} \gamma_{A}^{\prime}=\gamma_{A}$, with a term $\delta=\gamma-\beta J(\alpha) \in \pi_{n+k}(P D / O)$ satisfying $j_{*} \delta=j_{*}(\gamma-\beta J(\alpha))=0$. Suppose $P^{k}$ is simply connected, so that each $d(i) \geqq 2$; since the terms $\gamma_{A}^{\prime}$ are in the image of $\pi_{n+|A|}\left(P L_{\tilde{d}(A)+1}\right)$, they may be successively removed by PL self-equivalences. Therefore, there is a PL selfequivalence $\psi_{2}$ of $S^{n} \times P^{k}$ such that

$$
0 \cdot \psi_{1} \psi_{2}=\delta \in \pi_{n+k}(P D / O)
$$

In other words, $\delta$ is in the inertia group of $S^{n} \times P^{k}$. Hence $\delta=0$ by Theorem A.

If $P^{k}$ is not simply connected, the above argument fails for terms $\gamma_{A}^{\prime}$ such that the product of all factors not in $A$ is a torus. However, if one takes repeated infinite cyclic coverings, it follows that such terms $\gamma_{A}^{\prime}$ must also vanish by Theorem A. Thus $\delta=0$ holds without the assumption that $P^{k}$ is simply connected.

Thus $f(\beta, \gamma)$ is homotopy equivalent to a smoothing whose $\pi_{n+k}(P D / O)$ term vanishes. The final step in the proof is to show that the $\pi_{n+k}(P D / O)$ components of homotopy equivalent smoothings of orientable manifolds cannot differ by an element of $\partial P_{n+k+1}$. This is a trivial exercise in the use of the principle given at the end of 2.2. Since the $\pi_{n+k}(P D / O)$ component of $f(\beta, \gamma)$ is $\gamma-\beta J(\alpha) \in \partial P_{n+k+1}$, this element must vanish, and the Lemma is proved.

4.2. In this subsection we discuss some consequences of the stable equivalence theorems due to Mazur in the simply connected case [19] and Milnor in the nonsimply connected case [20]. If $M^{n}$ is any closed $\pi$-manifold with vanishing Whitehead group and $h$ is an oriented homotopy self-equivalence of $M$, then there is an orientation-preserving diffeomorphism $H: M \times S^{n+k} \rightarrow M \times S^{n+k}$ for all $k \geqq 3$ such that $\pi_{M} H \mid M=h$. Actually, it can be shown that $H$ is homotopic to a fiber 
homotopy equivalence covering $h$, and this fiber homotopy equivalence in $[M, G]$ actually represents the normal invariant of $q(h)$ in $[M, G / O]$ (see [3] for this interpretation of the normal invariant, and compare with [23]).

4.3. Proof of Theorem C. As stated before, we assume $k \leqq n-4$. For by 2.1, if $k \geqq n-3$ we know that $I\left(S_{\beta}^{n} \times P^{k}\right)=0$ for all $P^{k}$. As noted in 2.1, the truth of Theorem $\mathrm{C}$ has already been established for many homotopy spheres if $k \geqq(n+1) / 2$ by the work of Novikov and Antonelli.

If $\varphi \in \mathscr{E}_{1}\left(S^{n} \times P^{n}\right)$ induces the map $h=\pi_{P} \varphi \mid P$ (which is necessarily a homotopy self-equivalence) we have shown above that a diffeomorphism $H$ of $S^{n} \times P^{n}$ may be constructed. Furthermore, up to homotopy we may write $\varphi=H \varphi_{0}$, where $\pi_{P} \varphi \mid P=1_{P}$. Hence if $H \varphi_{0}(\beta, \gamma)=(\beta, 0)$-equivalently, $\varphi_{0}(\beta, \gamma)=H^{-1}(\beta, 0)$-we wish to compute the $\pi_{n+k}(G / O)$ factors on each side of this equation. Now $h$ is a product of fiber homotopy equivalences $h_{i}$ and $H$ is a product of associated diffeomorphisms $H_{i}$, and by the formula due to Novikov appearing in 2.2(ii) we may assume that the induced fiber homotopy equivalence is the stabilization of the inverse of $h_{i}$. A routine computation now shows that the right-hand side will have no $\pi_{n+k}(P D / O)$ factor, and hence none exists for $G / O$ either.

To compute the left-hand side, we proceed in much the same manner as in 4.1. We again show $j_{*}(\gamma-\beta J(\alpha))=0, \varphi_{0}$ is homotopic to a PL homeomorphism, and ultimately $\gamma-\beta J(\alpha)$ is shown to be the $\pi_{n+k}(P D / O)$ component of the right-hand side. By the reasoning of 4.1, this class vanishes, and the result is proved.

Appendix. Derivation of formulas in 3.3. We derive formulas 3.3(i)-(iii) for two reasons. First of all, generalizations of them are used in the proof of Theorem C. Secondly, they have been used subsequently by the author to investigate the plumbing pairing. It should be noted that the action of a class $g_{A} \in \pi_{|A|}\left(S O_{n+1}\right) \subseteq$ $\left[T^{k}, S O_{n+1}\right]$ (see 3.3) only depends on its image in $\pi_{|A|}\left(G_{n+1}\right)$. Therefore, we shall work with $\left[T^{k}, G_{n+1}\right]$ and in the process obtain the generalizations necessary for Theorem $\mathrm{C}$. The only property of $P D / O$ used is that it is a highly homotopy associative and commutative $H$-space. Hence we can make the argument for $H$, where $H$ is $P D / O, G / O$, or $T o p / O$ [4].

Derivation of (i). Let $T_{A} \subseteq T^{k}$ be the substances corresponding to the subset $A \subseteq\{1, \ldots, k\}$, and let $S_{A}$ be the sphere of the same dimension. The map $g_{A}$ is related to a fiber homotopy self-equivalence of $T_{A} \times S^{n}$ which passes to a map $h$ on $S_{A} \times S^{n}$ via the collapsing map $T_{A} \rightarrow S_{A}$. Therefore it suffices to look at $\left[S^{n} \times S_{A}, H\right.$ ], which maps monomorphically into $\left[S^{n} \times T_{A}, H\right]$ and $\left[S^{n} \times T^{k}, H\right]$ via composition with projections.

The basic idea is to exploit the natural isomorphism $[X, H] \cong[S X, B H]$, where addition is derivable from the $H$-space $B H$. Now the suspension of $S^{n} \times S_{A}$ is the wedge of $S^{n+1}, S S_{A}$, and $S\left(S^{n} S_{A}\right)$, and hence the object is to compute the restrictions of $S(\pi h): S\left(S^{n} \times S_{A}\right) \rightarrow S^{n+1}$ to the three summands. But the restriction to $S^{n+1}$ is the suspension of the identity ( $h$ is fiber-preserving and basepoint-preserving). The map $S S_{A} \rightarrow S^{n+1}$ is the suspended evaluation map of $h$, which vanishes 
provided $k<n$. Finally, the map $S\left(S^{n} S_{A}\right) \rightarrow S^{n+1}$ is induced by the Hopf construction; in particular, if $g_{A}$ comes from $\left[T_{A}, S O_{n+1}\right]$, then the homotopy class of this map is $J\left(g_{A}\right)$. Hence the components of $S(\pi h)^{*} \alpha_{n}$ are $\alpha_{n}\left(S^{n+1}\right.$ factor), $O\left(S_{A}\right.$ factor), and $\alpha_{n} \cdot J\left(g_{A}\right)\left(S^{n+1} S_{A}\right.$ factor).

Derivation of (ii). Let $T_{A}, T_{B}$ be the subtori corresponding to the disjoint subsets $A, B$. The induced fiber homotopy equivalence of $S^{n} \times T_{A} \times T_{B}$ over $T_{A}$ passes to a fiber homotopy equivalence of $S^{n} S_{B} \times S_{A}$ over $S_{A}$. The remainder of the derivation is nearly identical to (i).

Derivation of (iii). Let $C=A-A \cap B, D=A \cap B$, and $E=B-A \cap B$; the cases where $C$ or $E$ may be empty are included; we make the convention that $S_{\varnothing}=\{\mathrm{pt}\}$. Here we obtain a fiber homotopy equivalence $h \times 1_{C}$ of $\left(S^{n} \times S_{C} \times S_{D}\right) \times S_{E}$ over $S_{C} \times S_{D}$ such that $h$ is the identity of the product of the positive hemispheres $D_{C}^{+}, D_{D}^{+} \subseteq S_{C}, S_{D}$.

We begin by considering the map $S(p h): S\left(S^{n} \times S_{C} \times S_{D}\right) \rightarrow S^{n+1} S_{D}$, where $p$ is projection onto $S^{n} \times S_{D}$ followed by collapse onto the smash product. Now the suspension of $S^{n} \times S_{C} \times S_{D}$ is a wedge of at most seven spheres, one for each nonempty subset of $\left\{S^{n}, S_{C}, S_{D}\right\}$; if $C=\varnothing$, then the summands corresponding to smash products involving $S_{C}$ do not appear. We next look at all possible restrictions of $S(p h)$ to summands of $S\left(S^{n} \times S_{C} \times S_{D}\right)$.

[a] $S^{n+1}$ and $S S_{D}$. The restriction of $S(p h)$ to these spheres is trivial since their dimension is less than that of $S^{n+1} S_{D}$.

[b] $S^{n+1} S_{C}$ and $S S_{C}$. Consider the composite

$$
S^{n} \times S_{C} \stackrel{(1, *)}{\longrightarrow} S^{n} \times S_{C} \times S_{D} \stackrel{\pi h}{\longrightarrow} S^{n} \times S_{D} \longrightarrow S^{n} S_{D}
$$

The projections of the composite of the first two mappings onto the $S^{n}$ and $S_{D}$ factors are easy to determine. In particular, the projection onto $S_{D}$ is constant. But if $\varphi: U \rightarrow V \times W$ is any map such that $\pi_{W} \varphi$ is constant then the composite into $V \wedge W$ is trivial provided everything is arcwise connected. Hence the restrictions of $S(p h)$ to $S^{n+1} S_{C}$ and $S S_{C}$ are trivial.

[c] $S^{n+1} S_{D}$. The degree of the restriction of $S(p h)$ to $S^{n+1} S_{D}$ may be computed from the cohomology of the spaces involved, and it is +1 .

[d] $S^{n+1}\left(S_{C} \wedge S_{D}\right)$. We claim this restriction is also homotopically trivial. First of all, $h$ extends to a map of $I \times S^{n} \times S_{C} \times S_{D}$ in the obvious fashion. Next observe that the restriction to $I \times S^{n} \times D_{\bar{D}} \times S_{C}$ is the identity. Hence $h$ extends further to a fiber-preserving map $h^{\prime}$ over $S_{C}$ on the union $I \times S^{n} \times S_{D} \times S_{C} \cup D^{n+1} \times D_{\bar{D}}^{-} \times S_{E}$, where $I \times S^{n} \subseteq D^{n+1}$ by the annulus embedding and the map is the identity on the second portion.

Now $S^{n} \times I \times S_{D} \cup D^{n+1} \times D_{D}^{+}$is a manifold with boundary $S^{n} \times S_{D} \cup S^{n} S_{D}$, and by restriction $h^{\prime}$ induces a fiber homotopy equivalence $h^{n}$ of $S^{n} S_{D} \times S_{C}$ on the other boundary component. If one chases a suitable family of diagrams involving the fiber homotopy equivalence on the cobordism, it follows that the restriction of $S(p h)$ to $S^{n+1}\left(S_{D} \wedge S_{C}\right)$ is homotopic to the Hopf construction on the map 
$S^{n} S_{D} \times S_{C} \rightarrow S^{n} S_{D}$ induced by the fiber homotopy equivalence $h^{\prime \prime}$. But on each fiber

$$
S^{n} S_{D}=S^{n} \times D_{D}^{+} \cup D^{n+1} \times \partial D_{D}^{+}
$$

the fiber homotopy equivalence maps $S^{n} \times D_{D}^{+}$and $D^{n+1} \times \partial D_{D}^{+}$to themselves, the latter by the identity. It then follows that the induced fiber homotopy equivalence of $S^{n} S_{D} \times S_{E}$ is fiber homotopic to the identity by the Alexander trick. Hence the Hopf construction is homotopically trivial.

We proceed to derive 3.3(iii). If $E=\varnothing$, then the result is immediate since the only summand of $S\left(S^{n} \times S_{C} \times S_{D}\right)$ on which $S(p h)$ is nontrivial is $S S_{D}$, and the map $S S_{D} \rightarrow S S_{D}$ is the identity by [c]. On the other hand, if $E \neq \varnothing$, then consider the smash product of $h$ with the identity on $S_{E}$. The summand $\pi_{n+|B|}(H)$ is in the image of the projection induced composite

$$
\begin{aligned}
{\left[S^{n} S_{D} \wedge S_{E}, H\right] } & \rightarrow\left[\left(S^{n} \times S_{D}\right) \wedge S_{E}, H\right] \rightarrow\left[\left(S^{n} \times S_{C} \times S_{D}\right) \wedge S_{E}, H\right] \\
& \rightarrow\left[S^{n} \times S_{C} \times S_{D} \times S_{E}, H\right],
\end{aligned}
$$

and therefore it suffices to compute the effect of the composition $h^{*}$ on an element $\alpha \in\left[S^{n} \times S_{C} \times S_{D}, \Omega^{|E|} H\right]$ which is in the image of $\pi_{n+|D|}\left(\Omega^{|E|} H\right)$. According to the factor by factor analysis of restrictions appearing in [a]-[d], we have $h^{*} \alpha=\alpha$ as desired.

\section{REFERENCES}

1. J. F. Adams, On the non-existence of elements of Hopf invariant one, Ann. of Math. (2) 72 (1960), 20-104. MR 25 \#4530.

2. P. L. Antonelli, On the stable diffeomorphism of homotopy spheres in the stable range, $n<2 p$, Bull. Amer. Math. Soc. 75 (1969), 343-346. MR 39 \#2174.

3. M. A. Armstrong, Lectures on the Hauptvermutung according to Lashof and Rothenberg, Institute for Advanced Study, Princeton, N. J., 1968 (mimeographed).

4. J. M. Boardman and R. M. Vogt, Homotopy-everything H-spaces, Bull. Amer. Math. Soc. 74 (1968), 1117-1122. MR 38 \#5215.

5. E. H. Brown, Jr. and F. P. Peterson, Whitehead products and cohomology operations, Quart. J. Math. Oxford Ser. (2) 15 (1964), 116-120. MR 28 \#4549.

6. R. DeSapio, Differential structures on a product of spheres. I, Comment. Math. Helv. 44 (1969), 61-69; II, Ann. of Math. (2) 89 (1969), 305-314. MR 39 \#4857; MR 39 \#7611.

7. M. W. Hirsch, On homotopy spheres of low dimension, Differential and Combinatorial Topology (A Symposium in Honor of M. Morse), Princeton Math. Series, no. 27, Princeton Univ. Press, Princeton, N. J., 1965, pp. 199-204. MR 31 \#4042.

8. W.-C. Hsiang, J. Levine, and R. H. Szczarba, On the normal bundle of a homotopy sphere in Euclidean space, Topology 3 (1965), 173-181. MR 30 \#5323.

9. W.-C. Hsiang and J. Shaneson, Fake tori, the annulus conjecture, and the conjectures of Kirby, Proc. Nat. Acad. Sci. U.S.A. 63 (1969), 687-691.

10. N. Jacobson, Lectures in abstract algebra. Vol. II. Linear algebra, Van Nostrand, Princeton, N. J., 1953. MR 14, 837.

11. M. Kato, A concordance classification of PL homeomorphisms of $S^{p} \times S^{q}$, Topology 8 (1969), 371-384.

12. K. Kawakubo, Smooth structures on $S^{p} \times S^{q}$, Proc. Japan Acad. 45 (1969), 215-218. MR 40 \#2107. 
13. M. A. Kervaire, Some nonstable homotopy groups of Lie groups, Illinois J. Math. 4 (1960), 161-169. MR 22 \#4075.

14. A. Kosinski, On the inertia group of $\pi$-manifolds, Amer. J. Math. 89 (1967), 227-248. MR 35 \#4936.

15. R. Lashof and M. Rothenberg, Microbundles and smoothing, Topology 3 (1965), 357-388. MR 31 \#752.

16. J. Levine, A classification of differentiable knots, Ann. of Math. (2) 82 (1965), $15-50$. MR 31 \#5211.

17. - Self-equivalences of $S^{n} \times S^{k}$, Trans. Amer. Math. Soc. 143 (1969), 523-543. MR 40 \#2098.

18. M. Mahowald, The metastable homotopy of $S^{n}$, Mem. Amer. Math. Soc. No. 72 (1967). MR 38 \#5216.

19. B. C. Mazur, Stable equivalence of differentiable manifolds, Bull. Amer. Math. Soc. 67 (1961), 377-384. MR 24 \#A557.

20. J. Milnor, Two complexes which are homeomorphic but combinatorially distinct, Ann. of Math. (2) 74 (1961), 575-590. MR 24 \#A2961; errata, MR 25, p. 1242.

21. S. P. Novikov, Homotopically equivalent smooth manifolds. I, Izv. Akad. Nauk SSSR Ser. Mat. 28 (1964), 365-474; English transl., Amer. Math. Soc. Transl. (2) 48 (1965), 271-396. MR 28 \#5445.

22. —— Differentiable sphere bundles, Izv. Akad. Nauk SSSR Ser. Mat. 29 (1965), 71-96;

English transl., Amer. Math. Soc. Transl. (2) 63 (1967), 217-244. MR 30 \#4266.

23. C. P. Rourke, The Hauptvermutung according to Sullivan, Institute for Advanced Study, Princeton, N. J., 1968 (mimeographed).

24. H. Sato, Diffeomorphism groups and classification of manifolds, J. Math. Soc. Japan 21 (1969), 1-36. MR 39 \#3525.

25. - Diffeomorphism groups of $S^{p} \times S^{q}$ and exotic spheres, Quart. J. Math. Oxford Ser. (2) 79 (1969), 255-276.

26. R. Schultz, Smooth structures on $S^{p} \times S^{q}$, Ann. of Math. (2) 90 (1969), 187-198. MR 40 \#3560.

27. _ Smoothings of sphere bundles over spheres in the stable range, Invent. Math. 9 (1969), 81-88.

28. J. L. Shaneson, Wall's surgery obstruction groups for $G \times Z$, Ann. of Math. (2) 90 (1969), 296-334. MR 39 \#7614.

29. D. Sullivan, Triangulating and smoothing homotopy equivalences and homeomorphisms, Geometric Topology Seminar Notes, Princeton University, Princeton, N. J., 1967 (mimeographed).

30. E. C. Turner, Diffeomorphisms of a product of spheres, Invent. Math. 8 (1969), 69-82. MR 40 \#3562.

31. C. T. C. Wall, Killing the middle homotopy groups of odd-dimensional manifolds, Trans. Amer. Math. Soc. 103 (1962), 421-433. MR 25 \#2621.

32. - Classification problems in differential topology. II. Diffeomorphisms of handlebodies, Topology 2 (1963), 263-272. MR 27 \#6278.

33. - Surgery of compact manifolds (to appear).

34. - On homotopy tori and the annulus theorem, Bull. London Math. Soc. 1 (1969), 95-97. MR 39 \#3498.

Purdue University, LAFAYETTE, INDIANA 47907 